This is the author's final, peer-reviewed manuscript as accepted for publication. The publisher-formatted version may be available through the publisher's web site or your institution's library.

\title{
Implicit function theorem via the DSM
}
A. G. Ramm

\section{How to cite this manuscript}

If you make reference to this version of the manuscript, use the following information:

Ramm, A. G. (2010). Implicit function theorem via the DSM. Retrieved from http://krex.ksu.edu

\section{Published Version Information}

Citation: Ramm, A.G. (2010). Implicit function theorem via the DSM. Nonlinear Analysis: Theory, Methods and Applications,72(3-4), 1916-1921.

Copyright: Copyright @ 2009 Elsevier Ltd all rights reserved.

Digital Object Identifier (DOI): doi:10.1016/j.na.2009.09.032

\section{Publisher's Link:}

http://www.elsevier.com/wps/find/journaldescription.cws_home/239/description\#descript ion 
A G Ramm, Implicit Function Theorem via the DSM, Nonlinear Analysis: Theory, Methods and Appl., 72, N3-4, (2010), 1916-1921. 


\title{
Implicit Function Theorem via the DSM
}

\author{
A G Ramm \\ Department of Mathematics \\ Kansas State University, Manhattan, KS 66506-2602, USA \\ ramm@math.ksu.edu
}

\begin{abstract}
Sufficient conditions are given for an implicit function theorem to hold. The result is established by an application of the Dynamical Systems Method (DSM). It allows one to solve a class of nonlinear operator equations in the case when the Fréchet derivative of the nonlinear operator is a smoothing operator, so that its inverse is an unbounded operator.
\end{abstract}

$M S C: 47 \mathrm{~J} 05,47 \mathrm{~J} 25$,

Key words: Dynamical Systems Method (DSM); Hard implicit function theorem; Newton's method

\section{Introduction}

The aim of this paper is to demonstrate the power of the Dynamical Systems Method (DSM) as a tool for proving theoretical results. The DSM was systematically developed in [6] and applied to solving nonlinear operator equations in [6] (see also [7]), where the emphasis was on convergence and stability of the DSM-based algorithms for solving operator equations, especially nonlinear and ill-posed equations. The DSM for solving an operator equation $F(u)=h$ consists of finding a nonlinear map $u \mapsto \Phi(t, u)$, depending on a parameter $t \in[0, \infty)$, that has the following three properties:

(1) the Cauchy problem

$$
\dot{u}=\Phi(t, u), \quad u(0)=u_{0} \quad\left(\dot{u}:=\frac{d u(t)}{d t}\right)
$$

has a unique global solution $u(t)$ for a given initial approximation $u_{0}$;

(2) the limit $u(\infty)=\lim _{t \rightarrow \infty} u(t)$ exists; and

(3) this limit solves the original equation $F(u)=h$, i.e., $F(u(\infty))=h$.

The operator $F: H \rightarrow H$ is a nonlinear map in a Hilbert space $H$. It is assumed that the equation $F(u)=h$ has a solution, possibly nonunique.

The problem is to find a $\Phi$ such that the properties (1), (2), and (3) hold. Various choices of $\Phi$ for which these properties hold are proposed in [6], where the DSM is justified for wide classes of operator equations, in particular, for 
some classes of nonlinear ill-posed equations (i.e., equations $F(u)=0$ for which the linear operator $F^{\prime}(u)$ is not boundedly invertible). By $F^{\prime}(u)$ we denote the Fréchet derivative of the nonlinear map $F$ at the element $u$.

In this note the DSM is used as a tool for proving a "hard" implicit function theorem.

Let us first recall the usual implicit function theorem. Let $U$ solve the equation $F(U)=f$.

Proposition: If $F(U)=f, F$ is a $C^{1}$-map in a Hilbert space $H$, and $F^{\prime}(U)$ is a boundedly invertible operator, i.e., $\left.\|\left[F^{\prime}(U)\right]^{-1}\right] \| \leq m$, then the equation

$$
F(u)=h
$$

is uniquely solvable for every $h$ sufficiently close to $f$.

For convenience of the reader we include a proof of this known result.

Proof of the Proposition. First, one can reduce the problem to the case $u=0$ and $h=0$. This is done as follows. Let $u=U+z, h-f=p$, $F(U+z)-F(U):=\phi(z)$. Then $\phi(0)=0, \phi^{\prime}(0)=F^{\prime}(U)$, and equation (1.1) is equivalent to the equation

$$
\phi(z)=p
$$

with the assumptions

$$
\phi(0)=0, \quad \lim _{z \rightarrow 0}\left\|\phi^{\prime}(z)-\phi^{\prime}(0)\right\|=0, \quad\left\|\left[\phi^{\prime}(0)\right]^{-1}\right\| \leq m .
$$

We want to prove that equation (1.2) under the assumptions (1.3) has a unique solution $z=z(p)$, such that $z(0)=0$, and $\lim _{p \rightarrow 0} z(p)=0$. To prove this, consider the equation

$$
z=z-\left[\phi^{\prime}(0)\right]^{-1}(\phi(z)-p):=B(z)
$$

and check that the operator $B$ is a contraction in a ball $\mathcal{B}_{\epsilon}:=\{z:\|z\| \leq \epsilon\}$ if $\epsilon>0$ is sufficiently small, and $B$ maps $\mathcal{B}_{\epsilon}$ into itself. If this is proved, then the desired result follows from the contraction mapping principle.

One has

$$
\|B(z)\|=\left\|z-\left[\phi^{\prime}(0)\right]^{-1}\left(\phi^{\prime}(0) z+\eta-p\right)\right\| \leq m\|\eta\|+m\|p\|,
$$

where $\|\eta\|=o(\|z\|)$. If $\epsilon$ is so small that $m\|\eta\|<\frac{\epsilon}{2}$ and $p$ is so small that $m\|p\|<\frac{\epsilon}{2}$, then $\|B(z)\|<\epsilon$, so $B: \mathcal{B}_{\epsilon} \rightarrow \mathcal{B}_{\epsilon}$.

Let us check that $B$ is a contraction mapping in $\mathcal{B}_{\epsilon}$. One has:

$$
\begin{aligned}
\|B z-B y\| & =\left\|z-y-\left[\phi^{\prime}(0)\right]^{-1}(\phi(z)-\phi(y))\right\| \\
& =\left\|z-y-\left[\phi^{\prime}(0)\right]^{-1} \int_{0}^{1} \phi^{\prime}(y+t(z-y)) d t(z-y)\right\| \\
& \leq m \int_{0}^{1}\left\|\phi^{\prime}(y+t(z-y))-\phi^{\prime}(0)\right\| d t\|z-y\| .
\end{aligned}
$$


If $y, z \in \mathcal{B}_{\epsilon}$, then

$$
\sup _{0 \leq t \leq 1}\left\|\phi^{\prime}(y+t(z-y))-\phi^{\prime}(0)\right\|=o(1), \quad \epsilon \rightarrow 0 .
$$

Therefore, if $\epsilon$ is so small that $m o(1)<1$, then $B$ is a contraction mapping in $\mathcal{B}_{\epsilon}$, and equation (1.2) has a unique solution $z=z(p)$ in $\mathcal{B}_{\epsilon}$, such that $z(0)=0$. The proof is complete.

The crucial assumptions, on which this proof is based, are assumptions (1.3).

Suppose now that $\phi^{\prime}(0)$ is not boundedly invertible, so that the last assumption in (1.3) is not valid. Then a theorem which still guarantees the existence of a solution to equation (1.2) for some set of $p$ is called a "hard" implicit function theorem. Examples of such theorems one may find, e.g., in [1], [2], [3], and [4].

Our goal in this paper is to establish a new theorem of this type using a new method of proof, based on the Dynamical Systems Method (DSM). In [8] we have demonstrated a theoretical application of the DSM by establishing some surjectivity results for nonlinear operators.

The result, presented in this paper, is a new illustration of the applicability of the DSM as a tool for proving theoretical results.

To formulate the result, let us introduce the notion of a scale of Hilbert spaces $H_{a}$ (see [5]). Let $H_{a} \subset H_{b}$ and $\|u\|_{b} \leq\|u\|_{a}$ if $a \geq b$. Example of spaces $H_{a}$ is the scale of Sobolev spaces $H_{a}=W^{a, 2}(D)$, where $D \subset \mathbb{R}^{n}$ is a bounded domain with a sufficiently smooth boundary.

Consider equation (1.1). Assume that

$$
F(U)=f ; \quad F: H_{a} \rightarrow H_{a+\delta}, \quad u \in B(U, R):=B_{a}(U, R),
$$

where $B_{a}(U, R):=\left\{u:\|u-U\|_{a} \leq R\right\}$ and $\delta=$ const $>0$, and the operator $F: H_{a} \rightarrow H_{a+\delta}$ is continuous. Furthermore, assume that $A:=A(u):=F^{\prime}(u)$ exists and is an isomorphism of $H_{a}$ onto $H_{a+\delta}$ :

$$
c_{0}\|v\|_{a} \leq\|A(u) v\|_{a+\delta} \leq c_{0}^{\prime}\|v\|_{a}, \quad u, v \in B(U, R),
$$

that

$$
\left\|A^{-1}(v) A(w)\right\|_{a} \leq c, \quad v, w \in B(U, R)
$$

and

$$
\left\|A^{-1}(u)[A(u)-A(v)]\right\|_{a} \leq c\|u-v\|_{a}, \quad u, v \in B(U, R) .
$$

Here and below we denote by $c>0$ various constants. Note that (1.8) implies

$$
\left\|A^{-1}(u) \psi\right\|_{a} \leq c_{0}^{-1}\|\psi\|_{a+\delta}, \quad \psi=A(u)[F(v)-h], \quad v \in B(U, R) .
$$

Assumption (1.8) implies that $A(u)$ is a smoothing operator similar to a smoothing integral operator, and its inverse is similar to the differentiation operator of order $\delta>0$. Therefore, the operator $A^{-1}(u)=\left[F^{\prime}(u)\right]^{-1}$ causes the "loss of the derivatives". In general, this may lead to a breakdown of the Newton process 
(method) in a finitely many steps. Our assumptions (1.7)-(1.10) guarantee that this will not happen.

Assume that

$$
u_{0} \in B_{a}(U, \rho), \quad h \in B_{a+\delta}(f, \rho),
$$

where $\rho>0$ is a sufficiently small number:

$$
\rho \leq \rho_{0}:=\frac{R}{1+c_{0}^{-1}\left(1+c_{0}^{\prime}\right)},
$$

and $c_{0}, c_{0}^{\prime}$ are the constants from (1.8). Then $F\left(u_{0}\right) \in B_{a+\delta}\left(f, c_{0}^{\prime} \rho\right)$, because $\left\|F\left(u_{0}\right)-F(U)\right\| \leq c_{0}^{\prime}\left\|u_{0}-U\right\| \leq c_{0}^{\prime} \rho$.

Consider the problem

$$
\dot{u}=-\left[F^{\prime}(u)\right]^{-1}(F(u)-h), \quad u(0)=u_{0} .
$$

Our basic result is:

Theorem 1.1. If the assumptions (1.7)-(1.11) hold, and $0<\rho \leq \rho_{0}:=$ $\frac{R}{1+c_{0}^{-1}\left(1+c_{0}^{\prime}\right)}$, where $c_{0}, c_{0}^{\prime}$ are the constants from (1.8), then problem (1.12) has a unique global solution $u(t)$, there exists $V:=u(\infty)$,

$$
\lim _{t \rightarrow \infty}\|u(t)-V\|_{a}=0
$$

and

$$
F(V)=h .
$$

Theorem 1.1 says that if $F(U)=f$ and $\rho \leq \rho_{0}$, then for any $h \in B_{a+\delta}(f, \rho)$ equation (1.1) is solvable and a solution to (1.1) is $u(\infty)$, where $u(\infty)$ solves problem (1.12).

In Section 2 we prove Theorem 1.1.

\section{Proof}

Let us outline the ideas of the proof. The local existence and uniqueness of the solution to (1.12) will be established if one verifies that the operator $A^{-1}(u)[F(u)-h]$ is locally Lipschitz in $H_{a}$. The global existence of this solution $u(t)$ will be established if one proves the uniform boundedness of $u(t)$ :

$$
\sup _{t \geq 0}\|u(t)\|_{a} \leq c .
$$

Let us first prove (in paragraph a) below) estimate (2.1), the existence of $u(\infty)$, and the relation (1.14), assuming the local existence of the solution to (1.12).

In paragraph b) below the local existence of the solution to (1.12) is proved.

a) If $u(t)$ exists locally, then the function

$$
g(t):=\|\phi\|_{a+\delta}:=\|F(u(t))-h\|_{a+\delta}
$$


satisfies the relation

$$
g \dot{g}=\left(F^{\prime}(u(t)) \dot{u}, \phi\right)_{a+\delta}=-g^{2},
$$

where equation (1.12) was used. Since $g \geq 0$, it follows from (2.3) that

$$
g(t) \leq g(0) e^{-t}, \quad g(0)=\left\|F\left(u_{0}\right)-h\right\|_{a+\delta} .
$$

From (1.12), (2.3) and (1.8) one gets:

$$
\|\dot{u}\|_{a} \leq \frac{1}{c_{0}}\|\phi\|_{a+\delta}=\frac{g(0)}{c_{0}} e^{-t}:=r e^{-t}, \quad r:=\frac{\left\|F\left(u_{0}\right)-h\right\|_{a+\delta}}{c_{0}} .
$$

Therefore,

$$
\lim _{t \rightarrow \infty}\|\dot{u}(t)\|_{a}=0
$$

and

$$
\int_{0}^{\infty}\|\dot{u}(t)\|_{a} d t<\infty
$$

This inequality implies

$$
\|u(\tau)-u(s)\| \leq \int_{s}^{\tau}\|\dot{u}(t) d t\|<\epsilon, \quad \tau>s>s(\epsilon),
$$

where $\epsilon>0$ is an arbitrary small fixed number, and $s(\epsilon)$ is a sufficiently large number. Thus, the limit $V:=\lim _{t \rightarrow \infty} u(t):=u(\infty)$ exists by the Cauchy criterion, and (1.13) holds. Assumptions (1.7) and (1.8) and relations (1.12), (1.13), and (2.6) imply (1.14).

Integrating inequality $(2.5)$ yields

$$
\left\|u(t)-u_{0}\right\|_{a} \leq r,
$$

and

$$
\|u(t)-u(\infty)\|_{a} \leq r e^{-t} .
$$

Inequality (2.8) implies (2.1).

b) Let us now prove the local existence of the solution to (1.12).

We prove that the operator in $(1.12) A^{-1}(u)[F(u)-h]$ is locally Lipschitz in $H_{a}$. This implies the local existence of the solution to (1.12).

One has

$$
\begin{aligned}
& \left\|A^{-1}(u)(F(u)-h)-A^{-1}(v)(F(v)-h)\right\|_{a} \leq\left\|\left[A^{-1}(u)-A^{-1}(v)\right](F(u)-h)\right\|_{a} \\
& +\left\|A^{-1}(v)(F(u)-F(v))\right\|_{a}:=I_{1}+I_{2} .
\end{aligned}
$$

Write

$$
F(u)-F(v)=\int_{0}^{1} A(v+t(u-v))(u-v) d t
$$


and use assumption (1.9) with $w=v+t(u-v)$ to conclude that

$$
I_{2} \leq c\|u-v\|_{a}
$$

Write

$$
A^{-1}(u)-A^{-1}(v)=A^{-1}(u)[A(v)-A(u)] A^{-1}(v),
$$

and use the estimate

$$
\left\|A^{-1}(v)[F(u)-h]\right\|_{a} \leq c,
$$

which is a consequence of assumptions (1.7) and (1.8). Then use assumption (1.10) to conclude that

$$
I_{1} \leq c\|u-v\|_{a} .
$$

From $(2.10),(2.12)$ and $(2.15)$ it follows that the operator $A^{-1}(u)[F(u)-h]$ is locally Lipschitz.

Note that

$$
\begin{array}{r}
\|u(t)-U\|_{a} \leq\left\|u(t)-u_{0}\right\|_{a}+\left\|u_{0}-U\right\|_{a} \leq r+\rho, \\
\|F(u(t))-h\|_{a+\delta} \leq\left\|F\left(u_{0}\right)-h\right\|_{a+\delta} \leq\left\|F\left(u_{0}\right)-f\right\|_{a+\delta}+\|f-h\|_{a+\delta} \leq\left(1+c_{0}^{\prime}\right) \rho,
\end{array}
$$

so, from (2.5) one gets

$$
r \leq \frac{\left(1+c_{0}^{\prime}\right) \rho}{c_{0}}
$$

Choose

$$
R \geq r+\rho .
$$

Then the trajectory $u(t)$ stays in the ball $B(U, R)$ for all $t \geq 0$, and, therefore, assumptions (1.7)-(1.10) hold in this ball for all $t \geq 0$.

Condition (2.19) and inequality (2.18) imply

$$
\rho \leq \rho_{0}=\frac{R}{1+c_{0}^{-1}\left(1+c_{0}^{\prime}\right)} .
$$

This is the "smallness" condition on $\rho$.

Theorem 1.1 is proved.

\section{Example}

Let

$$
F(u)=\int_{0}^{x} u^{2}(s) d s, \quad x \in[0,1]
$$

Then

$$
A(u) q=2 \int_{0}^{x} u(s) q(s) d s .
$$

Let $f=x$ and $U=1$. Then $F(U)=x$. Choose $a=1$ and $\delta=1$. Denote by $H_{a}=H_{a}(0,1)$ the usual Sobolev space. Assume that

$$
h \in B_{2}(x, \rho):=\left\{h:\|h-x\|_{2} \leq \rho\right\},
$$


and $\rho>0$ is sufficiently small. One can verify that

$$
A^{-1}(u) \psi=\frac{\psi^{\prime}(x)}{2 u(x)}
$$

for any $\psi \in H_{1}$.

Let us check conditions (1.7)-(1.11) for this example.

Condition (1.7) holds, because if $u_{n} \rightarrow u$ in $H_{1}$, then

$$
\int_{0}^{x} u_{n}^{2}(s) d s \rightarrow \int_{0}^{x} u^{2}(s) d s
$$

in $H_{2}$. To verify this, it is sufficient to check that

$$
\frac{d^{2}}{d x^{2}} \int_{0}^{x} u_{n}^{2}(s) d s \rightarrow 2 u u^{\prime},
$$

where $\rightarrow$ means the convergence in $H:=H_{0}:=L^{2}(0,1)$. In turn, this is verified if one checks that $u_{n}^{\prime} u_{n} \rightarrow u^{\prime} u$ in $L^{2}(0,1)$, provided that $u_{n}^{\prime} \rightarrow u^{\prime}$ in $L^{2}(0,1)$.

One has

$$
I_{n}:=\left\|u_{n}^{\prime} u_{n}-u^{\prime} u\right\|_{0} \leq\left\|\left(u_{n}^{\prime}-u^{\prime}\right) u_{n}\right\|_{0}+\left\|u^{\prime}\left(u_{n}-u\right)\right\|_{0} .
$$

Since $\left\|u_{n}^{\prime}\right\|_{0} \leq c$, one concludes that $\left\|u_{n}\right\|_{L^{\infty}(0,1)} \leq c_{1}$ and $\lim _{n \rightarrow \infty}\left\|u_{n}-u\right\|_{L^{\infty}}=$ 0 . Thus,

$$
\lim _{n \rightarrow \infty} I_{n}=0 .
$$

Condition (1.8) holds because $\|u\|_{L^{\infty}(0,1)} \leq c\|u\|_{1}$, and

$$
\left\|\int_{0}^{x} u(s) q(s) d s\right\|_{2} \leq c\left\|u^{\prime} q+u q^{\prime}\right\|_{0} \leq c\left(\|q\|_{L^{\infty}(0,1)}\|u\|_{1}+\|u\|_{L^{\infty}(0,1)}\|q\|_{1}\right),
$$

so

$$
\left\|\int_{0}^{x} u(s) q(s) d s\right\|_{2} \leq c_{0}^{\prime}\|u\|_{1}\|q\|_{1},
$$

and

$$
\left\|\int_{0}^{x} u q d s\right\|_{2} \geq\|u q\|_{1} \geq c_{0}\|q\|_{1},
$$

provided that $u \in B_{1}(1, \rho)$ and $\rho>0$ is sufficiently small.

Condition (1.9) holds because

$$
\left\|A^{-1}(v) A(w) q\right\|_{1}=\left\|\frac{1}{v(x)} w(x) q\right\|_{1} \leq c\|q\|_{1},
$$

provided that $u, w \in B_{1}(1, \rho)$ and $\rho>0$ is sufficiently small.

Condition (1.10) holds because

$$
\left\|A^{-1}(u) \int_{0}^{x}(u-v) q d s\right\|_{1}=\left\|\frac{u-v}{2 u} q\right\|_{1} \leq c\|u-v\|_{1}\|q\|_{1},
$$


provided that $u, v \in B_{1}(1, \rho)$ and $\rho>0$ is sufficiently small.

By Theorem 1.1 the equation

$$
F(u):=\int_{0}^{x} u^{2}(s) d s=h,
$$

where $\|h-x\|_{2} \leq \rho$ and $\rho>0$ is sufficiently small, has a solution $V$,

$$
F(V)=h .
$$

This solution can be obtained as $u(\infty)$, where $u(t)$ solves problem (1.12) and conditions (1.11) and (2.20) hold. 


\section{References}

[1] S.Alinhac and P. Gerard, Pseudo-differential Operators and the NashMoser Theorem, Amer. Math. Soc., Providence, RI, 2007.

[2] M. Berger, Nonlinearity and Functional Analysis, Academic Press, New York, 1977

[3] K.Deimling, Nonlinear Functional Analysis, Springer Verlag, Berlin, 1985.

[4] L.Hörmander, On the Nash-Moser implicit function theorem, Ann. Acad. Sci. Fenn. Math., Series A.I. Mathematics, 10, (1985), 255-259.

[5] S. Krein, Yu. Petunin, E. Semenov, Interpolation of Linear Operators, Amer. Math. Soc., Providence, RI, (1982).

[6] A. G. Ramm, Dynamical Systems Method for Solving Operator Equations, Elsevier, Amsterdam, 2007.

[7] A. G. Ramm, Dynamical systems method (DSM) and nonlinear problems, in Spectral Theory and Nonlinear Analysis, J. Lopez-Gomez, ed., World Scientific, Singapore, 2005, 201-228.

[8] A. G. Ramm, Dynamical systems method and a homeomorphism theorem, Amer. Math. Monthly, 113, N10, (2006), 928-933. 